\title{
Psychiatric Comorbidities Affect the Hospitalization Course of Parkinson's Disease Patients: A Cross-Sectional Inpatient Study
}

Vaishalee Namdev ${ }^{1}$, Goher Haneef ${ }^{2,}{ }^{3}$, Asma T. Khan ${ }^{4}$, Sayeda A. Basith ${ }^{5}$, Anuj Virani ${ }^{6}$, Johanna S. Canenguez Benitez ${ }^{7}$, Albulena Sejdiu ${ }^{8}$, Keerthika Mathialagan ${ }^{9}$, Pradipta Majumder 10,11

1. Medicine and Surgery, Mahatma Gandhi Memorial Medical College, Indore, IND 2. Internal Medicine, University of Health Sciences, Lahore, PAK 3. Emergency Medicine, University of Cincinnati Medical Center, Cincinnati, USA 4. Internal Medicine, Larkin Community Hospital, South Miami, USA 5. Psychiatry and Behavioral Sciences, Medical University of the Americas, Charlestown, KNA 6. Family Medicine, Windsor University School of Medicine, Cayon, KNA 7. Internal Medicine, Larkin Community Hospital, Houston, USA 8. Psychiatry, Saints Cyril and Methodius Hospital, Kumanovo, MKD 9. Psychiatry, Sree Balaji Medical College and Hospital, Chennai, IND 10. Psychiatry, Drexel University College of Medicine, Philadelphia, USA 11. Psychiatry, WellSpan Health, York, USA

Corresponding author: Vaishalee Namdev, vaishaleen9@gmail.com

\section{Abstract}

\section{Objectives}

We aim to delineate the differences in demographic characteristics and hospitalization outcomes including the severity of illness, hospitalization length of stay (LOS) and cost, utilization of deep brain stimulation (DBS), and disposition in Parkinson's disease (PD) inpatients with psychiatric comorbidities versus without psychiatric comorbidities.

\section{Methods}

We conducted a cross-sectional study using the Nationwide Inpatient Sample (NIS), included 56,844 PD inpatients (age $\geqslant 40$ years), and subdivided them by inpatients into those without psychiatric comorbidities $(\mathrm{N}=38,629)$ and with psychiatric comorbidities $(\mathrm{N}=18,471)$. We compared the distributions of demographic characteristics and hospitalization outcomes (severity of illness, utilization of DBS, and disposition) by performing Pearson's chi-square test, and we measured the differences in continuous variables (i.e., age, LOS, and cost) by using the independent samples t-test.

\section{Results}

A significantly higher proportion of PD inpatients with psychiatric comorbidities were female (44.4\%) and white (83\%) and had a moderate loss of functioning (48.8\%) compared to those without psychiatric comorbidities. PD inpatients with psychiatric comorbidities had an increased mean LOS (4.7 days vs. 3.7 days, $\mathrm{P}<0.001)$ but a lower mean cost $(\$ 37,445$ vs. $\$ 41,957, \mathrm{P}<0.001)$. Also, there was a significantly lower utilization of DBS in PD inpatients with psychiatric comorbidities (19.2\% vs. $26.9 \%, \mathrm{P}<0.001)$ compared to those without psychiatric comorbidities, and an adverse disposition of transfer to a skilled nursing facility/intermediate care facility $(47.1 \%$ vs. $39.6 \%$, P <0.001) compared to PD inpatients without psychiatric comorbidities.

\section{Conclusion}

Although PD patients with psychiatric comorbidities had a moderate loss of functioning, there was significant underutilization of DBS. Meanwhile, psychiatric comorbidities among PD patients led to increased LOS and transfer to skilled facilities.

Categories: Neurology, Psychiatry

Keywords: parkinsons disease, affective disorders, depression, cost of hospitalization, extended hospitalization stay, suicidal behavior

\section{Introduction}

Parkinson's disease (PD) is a progressive neurodegenerative disease with a range of motor and non-motor symptoms. According to the Parkinson's Foundation, nearly one million people live with PD in the United States (US), and almost 60,000 new cases of PD are diagnosed every year [1]. The disease appears to disproportionately affect men, who are 1.5 times more likely to have the disease than females. The incidence of PD increases with age. Even though PD is a disease of the elderly, close to 4\% of PD cases are younger than 50 years of age [1]. PD is more prevalent among Whites and Hispanics, followed by Asians and Blacks $[2]$. 
Patients with PD are susceptible to various comorbid neuropsychiatric conditions. Our study explores psychiatric comorbidities in PD. Common psychiatric comorbidities found in PD are depression, anxiety, and psychosis [3]. Some of the other common psychiatric and behavioral disorders include cognitive impairment, impulse control disorders, dysthymia, sleep, and sexual disorders [4]. Additionally, with the long-term use of pharmacological treatment, delirium and psychosis are also becoming more common. Mood disorders, especially depression, often predate the development of motor symptoms in PD [3].

A systematic review demonstrated that nearly half of the patient population with PD experiences depressive symptoms [5]. Recent studies have shown that the frequency of major depression is 7-19\%. Meanwhile, 10$30 \%$ of patients with PD have subtle forms of depression [5, 6]. Risk factors for depression in PD patients include female sex, a personal or family history of depression, early-onset of disease, "atypical" parkinsonism, and other psychiatric comorbidities [6]. A recent meta-analysis has found out that the point prevalence of any anxiety disorder is $31 \%$. Generalized anxiety disorder (14.1\%) is the most common form of anxiety disorder, followed by social phobia (13.8\%) and anxiety disorder not otherwise specified (13.3\%) [7]. There are four classes of affective symptoms in PD patients: individuals with a low probability of affective symptoms (60.4\%), individuals with anxiety alone (22\%), individuals with anxiety and co-existing depressive symptoms (8.6\%), and prominent depressive symptoms without anxiety (9\%) [8]. A recent study has shown that up to $40 \%$ of patients with PD experience generalized anxiety disorder, panic attacks, and social phobia [6]. The prevalence of apathy and emotional lability in patients with PD is $40 \%$ and $10 \%$, respectively [6].

The pathophysiology of these neuropsychiatric symptoms is multifaceted and includes alterations in the monoaminergic system. The noradrenergic and serotonergic circuits, as well as the degeneration of dopaminergic neurons, are commonly implicated in PD patients [9]. Unlike depression, the symptoms of mania and hypomania are directly associated with deep brain stimulation (DBS) [10]. They are mediated through the anterior cingulate cortex (ACC) and its functional connectivity with the substantia nigra [11]. Anxiety in PD is often a result of a pathological process rather than a response to disability. However, in social anxiety disorder (SAD), there is a positive correlation with increased density of dopamine transporters (DAT) compared to patients without SAD, thereby suggesting the role of dopamine dysfunction in this subset of patients [12].

Psychiatric comorbidities in PD patients are often associated with faster cognitive and motor decline, increased mortality, and caregiver burnout. Depression is one of the most significant determinants in the deterioration of quality of life (QoL), followed by anxiety, apathy, aggression, and irritability [13]. Higher rates of institutionalization, faster progression to dementia, and rapid motor decline are seen in PD patients with comorbid depression compared to patients without depression. There is a higher risk of suicide in patients who have PD with psychiatric comorbidities [14]. Poor adherence to antidepressants in patients with comorbid mood disorders has also increased all-cause mortality [15]. Early recognition and treatment of psychiatric comorbidities in PD could significantly improve the QoL, reduce the morbidity and mortality associated with the disease, and alleviate the substantial financial burden on the healthcare system [13]. The goal of our study is to delineate the differences in demographic characteristics and hospitalization outcomes, including the severity of illness, length of stay (LOS) and cost, utilization of DBS, and disposition in hospitalized PD patients by psychiatric comorbidities.

\section{Materials And Methods \\ Study sample}

We conducted a cross-sectional retrospective study using the Nationwide Inpatient Sample (NIS). The NIS is the largest inpatient database and represents a sample of 4,411 non-federal community hospitals across 44 states in the United States [16]. In this study, we included 56,844 patients (age $\geqslant 40$ years) with a primary discharge diagnosis ( $\mathrm{dx} 1$ variable in the NIS) of PD. The sample was further subdivided into two groups: PD inpatients without psychiatric comorbidities $(\mathrm{N}=38,629)$ versus with psychiatric comorbidities $(\mathrm{N}=18,471)$. The International Classification of Diseases, Ninth Revision, Clinical Modification (ICD-9-CM) diagnostic codes for PD and psychiatric comorbidities are shown in Table 1. 


\section{Cureus}

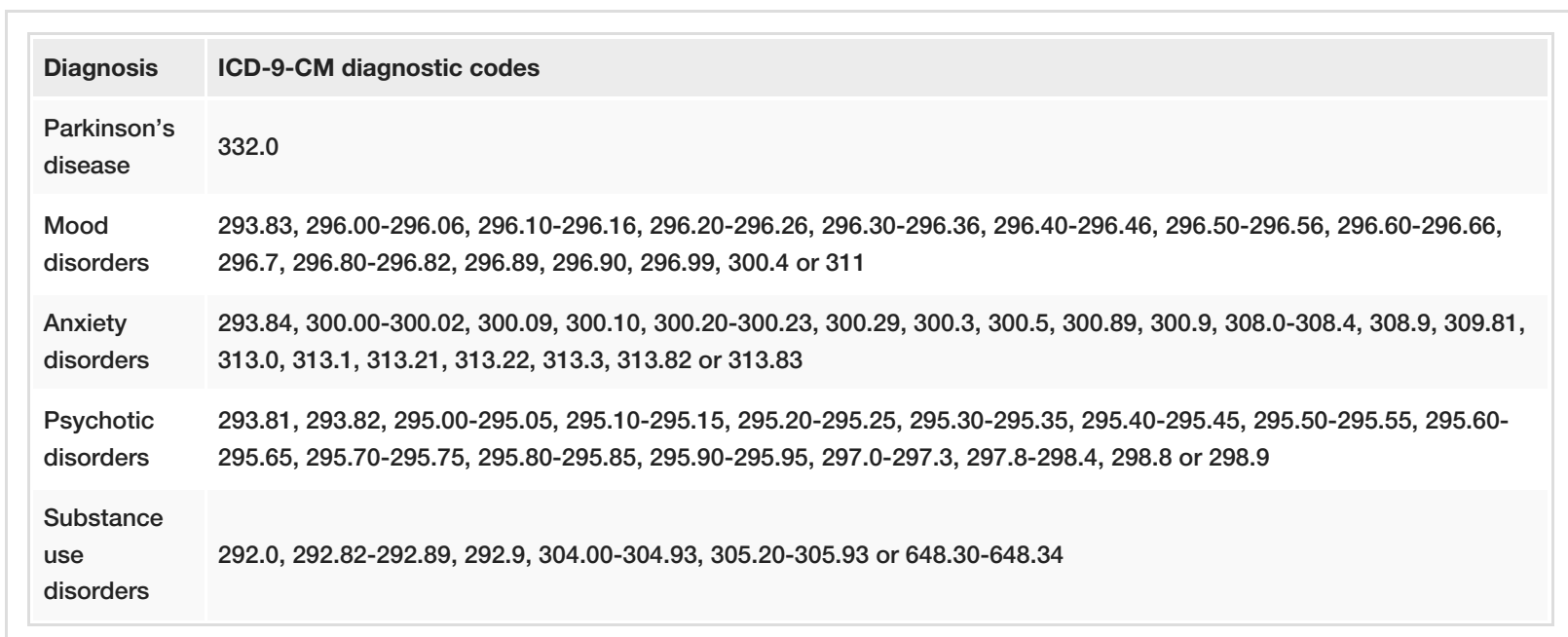

TABLE 1: Diagnostic codes used in the study sample

ICD-9-CM: International Classification of Diseases, Clinical Modification

\section{Variables}

Psychiatric comorbidities were defined by various co-diagnoses in the patient's records. We included mood disorders, anxiety disorders, psychotic disorders, and substance use disorders (SUD) as the entities of interest [17]. We calculated the LOS as the number of nights the patient was hospitalized for the management of primary discharge diagnosis ( $\mathrm{dx} 1$ ) of PD. We also included the total cost during hospitalization, excluding professional fees and non-covered charges. The hospitalization outcomes of interest included in this study are the severity of illness, which measures the loss of body functions (minor, moderate, or major) using the All-Patient Refined Diagnosis-Related Groups (DRGs) software developed by Health Information Systems (3M ${ }^{\mathrm{TM}}$ APR DRG Software, Health Information Systems, Salt Lake City, UT), and utilization of DBS (identified using ICD-9-CM procedure code 02.93), disposition status (routine, transfer to short-term hospital, transfer to skilled nursing facility/intermediate care facility (SNF/ICF), home health care, against medical advice and died) [18].

\section{Statistical analysis}

We compared the distributions of demographic characteristics and hospitalization outcomes (severity of illness, utilization of DBS, and disposition) in PD inpatients without versus with psychiatric comorbidities by performing descriptive statistics and Pearson's chi-square test. Next, we measured the differences in continuous variables (i.e., age, LOS, and cost) between PD inpatients without versus with psychiatric comorbidities using the independent sample t-test. All analyses were conducted using the Statistical Package for the Social Sciences (SPSS) version 26.0 (IBM Corp., Armonk, NY) and statistical significance was set at a two-sided $\mathrm{P}$ value of $<0.05$.

\section{Ethical approval}

The NIS is publicly available de-identified data that protects patients, physicians, and hospital-related information; hence, we were not required to take institutional review board permission for our study [16].

\section{Results}

Our study included 56,844 PD inpatients, and 18,471 inpatients (32.5\%) had psychiatric comorbidities. Among psychiatric comorbidities, a higher proportion of PD inpatients had mood disorders (72.7\%) and anxiety disorders (34.3\%) followed by psychotic disorders (12.4\%) and SUD (6\%) as shown in Figure 1. 


\section{Cureus}

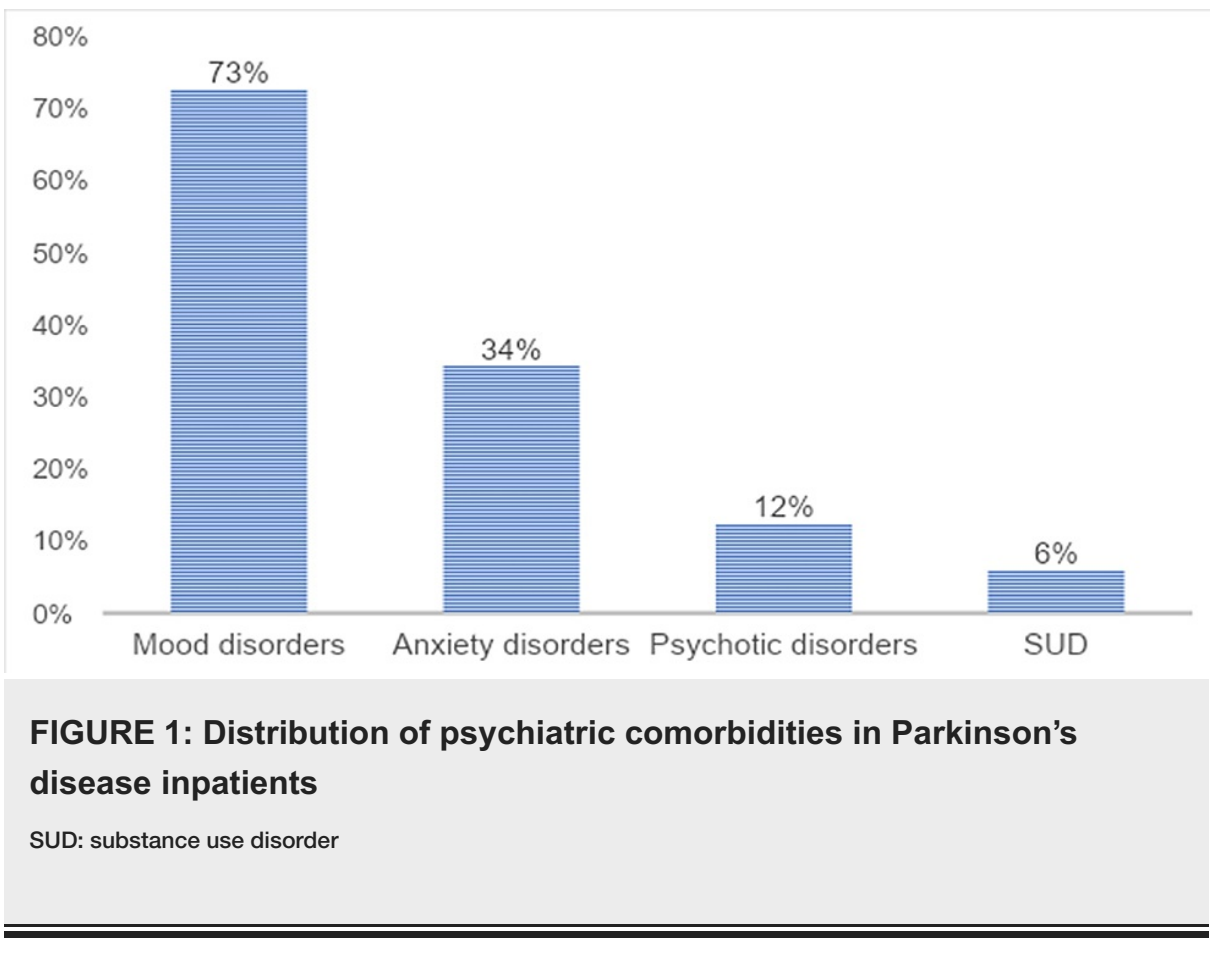

The mean age of PD inpatients with psychiatric comorbidities was 70.7 years. A significantly higher proportion of PD inpatients with psychiatric comorbidities were female (44.4\%) and white (83\%) compared to PD inpatients without psychiatric comorbidities. There was statistically no significant difference between the groups based on the median household income $(\mathrm{P}=0.150)$.

A higher proportion of PD inpatients with psychiatric comorbidities had a moderate loss of functioning ( $48.8 \%$ vs. $42.2 \%, \mathrm{P}<0.001)$, and the mortality rate was lower $(0.8 \%$ vs. $1.4 \%, \mathrm{P}<0.001)$ compared to PD inpatients without psychiatric comorbidities. PD inpatients with psychiatric comorbidities had an increased mean LOS ( 4.7 days vs. 3.7 days, $\mathrm{P}<0.001$ ) but a lower mean cost ( $\$ 37445$ vs. $\$ 41957, \mathrm{P}<0.001)$. There was a significantly lower utilization of DBS in PD inpatients with psychiatric comorbidities (19.2\% vs. $26.9 \%$, P $<0.001)$ compared to those without psychiatric comorbidities.

A significantly higher proportion of PD inpatients with psychiatric comorbidities were transferred to SNF/ICF (47.1\% vs. 39.6\% in PD inpatients without psychiatric comorbidities), as shown in Table 2. 


\section{Cureus}

\begin{tabular}{|c|c|c|c|}
\hline \multirow{2}{*}{ Variable } & \multicolumn{2}{|c|}{ Psychiatric comorbidities } & \multirow{2}{*}{$P$ value } \\
\hline & no & yes & \\
\hline Number of inpatients & 38629 & 18471 & - \\
\hline Mean age (SD), in years & $72.8(10.9)$ & $70.7(10.8)$ & $<0.001$ \\
\hline \multicolumn{4}{|l|}{ Sex, in \% } \\
\hline Male & 66.4 & 55.6 & \multirow{2}{*}{$<0.001$} \\
\hline Female & 33.6 & 44.4 & \\
\hline \multicolumn{4}{|l|}{ Race, in \% } \\
\hline White & 79.4 & 83.0 & \multirow{4}{*}{$<0.001$} \\
\hline Black & 7.5 & 4.7 & \\
\hline Hispanic & 6.6 & 7.1 & \\
\hline Others & 6.5 & 5.2 & \\
\hline \multicolumn{4}{|l|}{ Median household income, in \% } \\
\hline $0-25^{\text {th }}$ percentile & 22.4 & 23.3 & \multirow{4}{*}{0.150} \\
\hline $26^{\text {th }}-50^{\text {th }}$ percentile & 25.1 & 24.3 & \\
\hline $51^{\text {st }}-75^{\text {th }}$ percentile & 25.3 & 25.4 & \\
\hline $76^{\text {th }}-100^{\text {th }}$ percentile & 27.2 & 27.0 & \\
\hline \multicolumn{4}{|l|}{ Severity of illness, in \% } \\
\hline Minor loss of function & 35.6 & 29.4 & \multirow{3}{*}{$<0.001$} \\
\hline Moderate loss of function & 42.2 & 48.8 & \\
\hline Major loss of function & 22.2 & 21.8 & \\
\hline \multicolumn{4}{|l|}{ Hospitalization outcomes } \\
\hline Mean length of stay (SD), in days & $3.7(6.3)$ & $4.7(9.8)$ & $<0.001$ \\
\hline Mean cost (SD), in \$ & 41957 (46161) & 37445 (38597) & $<0.001$ \\
\hline Utilization of DBS, in \% & 26.9 & 19.2 & $<0.001$ \\
\hline \multicolumn{4}{|l|}{ Disposition } \\
\hline Routine & 43.7 & 36.1 & \multirow{5}{*}{$<0.001$} \\
\hline Transfer to SNF/ICF & 40.6 & 48.6 & \\
\hline Home health care & 13.8 & 14.1 & \\
\hline Against medical advice & 0.4 & 0.3 & \\
\hline Died & 1.4 & 0.8 & \\
\hline
\end{tabular}

\section{TABLE 2: Differences in Parkinson's disease inpatients by psychiatric comorbidities}

SD: standard deviation; DBS: deep brain stimulation; SNF: skilled nursing facility; ICF: intermediate care facility

\section{Discussion}

In our study, $32.5 \%$ of the patients with PD had psychiatric comorbidity, of which $72.7 \%$ had symptoms of mood disorders, followed by anxiety disorders (34.3\%). Symptoms of depression and anxiety could be early prodromal manifestations of PD [19]. Risk factors for depression in PD include female sex, personal and familial history of depression, early-onset PD, "atypical" parkinsonism, and other psychiatric comorbidities 
[6]. One of the biggest hurdles in diagnosing comorbid depression in PD is the overlap between the psychosomatic symptoms of depression and PD. The national institute of health recommends an inclusive approach, where the somatic symptoms are considered a part of depression [20]. Anxiety is often comorbid with depression in patients with PD. The most common manifestation in patients with PD is a generalized anxiety disorder, followed closely by panic disorder and social phobias, which reduce QoL [6, 21]. PD is also associated with situational phobias related to motor symptoms, for instance, freezing of gait and tremors in social situations [21].

Subsequently, we also found a higher prevalence of psychotic disorders and SUD among PD patients in our study. Some of the risk factors for psychosis in PD patients are advanced age, longer disease duration, advanced disease, disorders of the sleep-wake cycle, excessive dopamine therapy, a high degree of cognitive impairment, and a family history of dementia [22, 23]. Before dopamine replacement therapy (DRT), the prevalence of psychotic symptoms in untreated PD was less than 10\% [24]. Psychosis is a crucial determinant of hospitalization and nursing home placement in patients with PD, which plays a significant part in increased caregiver burnout. Repeated hospitalization also leads to a disruption in the patient's medication schedule leading to accelerated cognitive decline [6]. Visual hallucinations are the most reported symptoms, followed by auditory, tactile, and even olfactory hallucinations [22, 23].

Substance-related addictions, commonly known as dopamine disorder syndrome (DDS), are common among PD patients who use higher doses of dopamine than required. Pathology of the dopaminergic circuit is common to both PD and substance use disorders. DDS is a neuropsychiatric behavioral syndrome, where a disturbance in the impulse control system leads to addictive behaviors. Current estimates show that substance-related addiction in PD is due to DDS, and the most common substances of abuse are apomorphine and levodopa [25].

In our study, PD patients with psychiatric comorbidities had a moderate loss of functioning. Our results are consistent with a previous study, which reported pronounced cognitive decline and deterioration in activities of daily living [26]. Although PD patients with psychiatric comorbidities had a moderate loss of functioning, our study found a significant underutilization of DBS. DBS has shown promising results in treating motor fluctuations in moderate to severe PD. Several factors could be implicated in the underutilization of DBS by patients like misinformation about indications, side effects and cost, and insurance coverage burden [27].

Our study showed increased mean LOS in patients with psychiatric comorbidities and higher transfer rates to SNF/ICF. Depression in PD is often associated with worse QoL, increased cognitive impairment, and caregiver burden, leading to increased transfer to skilled facilities and an increased mean LOS [28]. Our study also found a lower mortality rate among PD inpatients with psychiatric comorbidities. Results from past studies on the effect of neuropsychiatric symptoms on mortality rates among PD patients have been conflicting. In addition to complications arising from PD, the progressive and degenerative nature of the disease by itself leads to increased mortality rates [29].

As a cross-sectional study, one of our limitations was that we could not find a causal relationship between the development of psychiatric comorbidities and the primary pathophysiology of PD. In addition to this, another limitation was that we used an administrative database and therefore lacked patient-level clinical information, as the patients were identified based on diagnostic codes rather than symptoms themselves, which can lead to underdiagnosis of the psychiatric comorbidities. We used the NIS, which enabled us to present data of a larger patient population, increasing the power of our study with minimal reporting bias.

\section{Conclusions}

PD patients with psychiatric comorbidities had a moderate loss of functioning, yet, there was significant underutilization of DBS. Psychiatric comorbidities led to extended hospitalization stays and higher disposition rates to nursing facilities. Further studies to assess the impact of psychiatric comorbidities in PD are essential for earlier diagnosis and management and to improve the QoL and cognitive functioning in this at-risk population.

\section{Additional Information \\ Disclosures}

Human subjects: Consent was obtained or waived by all participants in this study. Animal subjects: All authors have confirmed that this study did not involve animal subjects or tissue. Conflicts of interest: In compliance with the ICMJE uniform disclosure form, all authors declare the following: Payment/services info: All authors have declared that no financial support was received from any organization for the submitted work. Financial relationships: All authors have declared that they have no financial relationships at present or within the previous three years with any organizations that might have an interest in the submitted work. Other relationships: All authors have declared that there are no other relationships or activities that could appear to have influenced the submitted work. 


\section{References}

1. Statistics. (2021). Accessed: April 18, 2021: https://www.parkinson.org/UnderstandingParkinsons/Statistics.

2. Van Den Eeden SK, Tanner CM, Bernstein AL, Fross RD, Leimpeter A, Bloch DA, Nelson LM: Incidence of Parkinson's disease: variation by age, gender, and race/ethnicity. Am J Epidemiol. 2003, 157:1015-22. 10.1093/aje/kwg068

3. Marsh L: Depression and Parkinson's disease: current knowledge. Curr Neurol Neurosci Rep. 2013, 13:409. 10.1007/s11910-013-0409-5

4. Grover S, Somaiya M, Kumar S, Avasthi A: Psychiatric aspects of Parkinson's disease . J Neurosci Rural Pract. 2015, 6:65-76. 10.4103/0976-3147.143197

5. Reijnders JS, Ehrt U, Weber WE, Aarsland D, Leentjens AF: A systematic review of prevalence studies of depression in Parkinson's disease. Mov Disord. 2008, 23:183-9; quiz 313. 10.1002/mds.21803

6. Weintraub D, Mamikonyan E: The neuropsychiatry of Parkinson disease: a perfect storm . Am J Geriatr Psychiatry. 2019, 27:998-1018. 10.1016/j.jagp.2019.03.002

7. Broen MP, Narayen NE, Kuijf ML, Dissanayaka NN, Leentjens AF: Prevalence of anxiety in Parkinson's disease: a systematic review and meta-analysis. Mov Disord. 2016, 31:1125-33. 10.1002/mds.26643

8. Brown RG, Landau S, Hindle JV, et al.: Depression and anxiety related subtypes in Parkinson's disease . J Neurol Neurosurg Psychiatry. 2011, 82:803-9. 10.1136/innp.2010.213652

9. Aarsland D, Påhlhagen S, Ballard CG, Ehrt U, Svenningsson P: Depression in Parkinson disease-epidemiology, mechanisms and management. Nat Rev Neurol. 2011, 8:35-47. 10.1038/nrneurol.2011.189

10. Kulisevsky J, Berthier ML, Gironell A, Pascual-Sedano B, Molet J, Parés P: Mania following deep brain stimulation for Parkinson's disease. Neurology. 2002, 59:1421-4. 10.1212/wnl.59.9.1421

11. Ulla M, Thobois S, Llorca PM, et al.: Contact dependent reproducible hypomania induced by deep brain stimulation in Parkinson's disease: clinical, anatomical and functional imaging study. J Neurol Neurosurg Psychiatry. 2011, 82:607-14. 10.1136/jnnp.2009.199323

12. McKinlay A, Grace RC, Dalrymple-Alford JC, Anderson T, Fink J, Roger D: A profile of neuropsychiatric problems and their relationship to quality of life for Parkinson's disease patients without dementia. Parkinsonism Relat Disord. 2008, 14:37-42. 10.1016/j.parkreldis.2007.05.009

13. Ravina B, Camicioli R, Como PG, Marsh L, Jankovic J, Weintraub D, Elm J: The impact of depressive symptoms in early Parkinson disease. Neurology. 2007, 69:342-7. 10.1212/01.wnl.0000268695.63392.10

14. Lee T, Lee HB, Ahn MH, Kim J, Kim MS, Chung SJ, Hong JP: Increased suicide risk and clinical correlates of suicide among patients with Parkinson's disease. Parkinsonism Relat Disord. 2016, 32:102-7. 10.1016/j.parkreldis.2016.09.006

15. Shoval G, Stubbs B, Balicer RD, et al.: Low adherence to antidepressants is associated with increased mortality in Parkinson disease patients. Parkinsonism Relat Disord. 2017, 43:92-6. 10.1016/j.parkreldis.2017.07.032

16. Overview of the National (Nationwide) Inpatient Sample (NIS) . (2021). Accessed: April 18, 2021: https://www.hcup-us.ahrq.gov/nisoverview.jsp.

17. Marsh L, Williams JR, Rocco M, Grill S, Munro C, Dawson TM: Psychiatric comorbidities in patients with Parkinson disease and psychosis. Neurology. 2004, 63:293-300. 10.1212/01.wnl.0000129843.15756.a3

18. The National (Nationwide) Inpatient Sample (NIS) description of data elements . (2021). Accessed: April 18, 2021: https://www.hcup-us.ahrq.gov/db/nation/nis/nisdde.jsp.

19. Jacob EL, Gatto NM, Thompson A, Bordelon Y, Ritz B: Occurrence of depression and anxiety prior to Parkinson's disease. Parkinsonism Relat Disord. 2010, 16:576-81. 10.1016/j.parkreldis.2010.06.014

20. Marsh L, McDonald WM, Cummings J, Ravina B: Provisional diagnostic criteria for depression in Parkinson's disease: report of an NINDS/NIMH work group. Mov Disord. 2006, 21:148-58. 10.1002/mds.20723

21. Dissanayaka NN, White E, O'Sullivan JD, Marsh R, Pachana NA, Byrne GJ: The clinical spectrum of anxiety in Parkinson's disease. Mov Disord. 2014, 29:967-75. 10.1002/mds.25937

22. Forsaa EB, Larsen JP, Wentzel-Larsen T, Goetz CG, Stebbins GT, Aarsland D, Alves G: A 12-year populationbased study of psychosis in Parkinson disease. Arch Neurol. 2010, 67:996-1001. 10.1001/archneurol.2010.166

23. Imran S, Patel RS, Onyeaka HK, et al.: Comorbid depression and psychosis in Parkinson's disease: a report of 62,783 hospitalizations in the United States. Cureus. 2019, 11:e5227. 10.7759/cureus.5227

24. Fénelon G, Goetz CG, Karenberg A: Hallucinations in Parkinson disease in the prelevodopa era . Neurology. 2006, 66:93-8. 10.1212/01.wnl.0000191325.31068.c4

25. Ceravolo R, Frosini D, Rossi C, Bonuccelli U: Spectrum of addictions in Parkinson's disease: from dopamine dysregulation syndrome to impulse control disorders. J Neurol. 2010, $257:$ :S276-83. 10.1007/s00415-0105715-0

26. Starkstein SE, Mayberg HS, Leiguarda R, Preziosi TJ, Robinson RG: A prospective longitudinal study of depression, cognitive decline, and physical impairments in patients with Parkinson's disease. J Neurol Neurosurg Psychiatry. 1992, 55:377-82. 10.1136/jnnp.55.5.377

27. Kim MR, Yun JY, Jeon B, Lim YH, Kim KR, Yang HJ, Paek SH: Patients' reluctance to undergo deep brain stimulation for Parkinson's disease. Parkinsonism Relat Disord. 2016, 23:91-4. 10.1016/j.parkreldis.2015.11.010

28. Patel RS, Makani R, Mansuri Z, Patel U, Desai R, Chopra A: Impact of depression on hospitalization and related outcomes for Parkinson's disease patients: a nationwide inpatient sample-based retrospective study. Cureus. 2017, 9:e1648. 10.7759/cureus.1648

29. Morgan JC, Currie LJ, Harrison MB, Bennett JP Jr, Trugman JM, Wooten GF: Mortality in levodopa-treated Parkinson's disease. Parkinsons Dis. 2014, 2014:426976. 10.1155/2014/426976 